\title{
TMEM16 scramblases thin the membrane to enable lipid scrambling
}

\author{
Alessio Accardi ( $\nabla$ ala2022@med.cornell.edu ) \\ Weill Cornell Medical College https://orcid.org/0000-0002-6584-0102 \\ Maria Falzone \\ Weill Cornell Medical College \\ Zhang Feng \\ Weill Cornell Medical College \\ Omar Alvarenga \\ Weill Cornell Medical College \\ Yangang Pang \\ Weill Cornell Medical College \\ Byoung Lee \\ Cornell University \\ Xiaolu Cheng \\ Cornell University https://orcid.org/0000-0002-2785-6488

\section{Eva Fortea} \\ Weill Cornell Medical College \\ Simon Scheuring \\ Weill Cornell Medicine https://orcid.org/0000-0003-3534-069X
}

\section{Article}

Keywords: TMEM16 scramblases, lipid scrambling, membrane thinning

Posted Date: October 8th, 2021

DOI: https://doi.org/10.21203/rs.3.rs-955726/v1

License: (c) (i) This work is licensed under a Creative Commons Attribution 4.0 International License. Read Full License

Version of Record: A version of this preprint was published at Nature Communications on May 11th, 2022. See the published version at https://doi.org/10.1038/s41467-022-30300-z. 


\section{TMEM16 scramblases thin the membrane to enable lipid scrambling}

Maria E. Falzone ${ }^{1,2}$, Zhang Feng ${ }^{1}$, Omar E. Alvarenga ${ }^{1,3}$, Yangang Pang ${ }^{1}$, ByoungCheol Lee ${ }^{1, \wedge}$, Xiaolu Cheng ${ }^{4}$, Eva Fortea ${ }^{1,3}$, Simon Scheuring ${ }^{1}$, Alessio Accardi ${ }^{1,2,4 *}$

1 Department of Anesthesiology, Weill Cornell Medical College; 2 Department of Biochemistry, Weill Cornell Medical College; 3 Physiology, Biophysics and Systems Biology Graduate Program, Weill Cornell Medical College; 4 Department of Physiology and Biophysics, Weill Cornell Medical College

* correspondence to: ala2022@med.cornell.edu

^ ByoungCheol Lee's present address: Neurovascular Unit Research Group, Korea Brain Research Institute (KBRI), Daegu 41062, Republic of Korea. 


\begin{abstract}
TMEM16 scramblases dissipate the plasma membrane lipid asymmetry to activate multiple eukaryotic cellular pathways. It was proposed that lipid headgroups move between leaflets through a membrane-spanning hydrophilic groove. Direct information on lipid-groove interactions is lacking. We report the $2.3 \AA$ resolution cryoEM structure of the $\mathrm{Ca}^{2+}$-bound afTMEM16 scramblase in nanodiscs showing how rearrangement of individual lipids at the open pathway results in pronounced membrane thinning. Only the groove's intracellular vestibule contacts lipids, and mutagenesis suggests scrambling does not entail specific protein-lipid interactions with the extracellular vestibule. Further, we find scrambling can occur outside a closed groove in thinner membranes and is inhibited in thicker membranes despite an open pathway. Our results show how afTMEM16 thins the membrane to enable scrambling and that an open hydrophilic pathway is not a structural requirement to allow rapid transbilayer movement of lipids. This mechanism could be extended to other scramblases lacking a hydrophilic groove.
\end{abstract}




\section{Introduction}

Biological membranes play a fundamental role in many cellular signaling pathways as they define the physical boundaries of cellular compartments and actively modulate the function of integral and membrane-associated proteins. In eukaryotic cells, the composition and distribution of the phospholipid constituents of the membrane is tightly regulated by the activity of a variety of dedicated enzymes, flipases, flopases and scramblases ${ }^{1}$. The headgroup asymmetry of the plasma membrane is established by the action of ATP-driven pumps which distribute phosphatidylethanolamine (PE) and phosphatidylserine (PS) to the inner leaflet and phosphatidylcholine (PC) to the outer leaflet ${ }^{1}$. Activated phospholipid scramblases dissipate this asymmetry and expose PS on the extracellular leaflet. This is critical for multiple signaling pathways, ranging from apoptosis to blood coagulation and cell-cell fusion ${ }^{1,2}$. There are two known families of scramblases, the $\mathrm{Ca}^{2+}$-activated TMEM16 ${ }^{3-5}$ and the caspase-activated Xkrelated (Xkr) proteins ${ }^{6}$. Lipid scrambling by the TMEM16's is of critical importance for a myriad of physiological processes, including blood coagulation, bone mineralization, membrane fusion and viral entry ${ }^{2,4,7}$. Dysregulation of TMEM16 scramblase activity can have disastrous consequences, as both gain- and loss- of function mutations have been associated with disorders of blood, brain, bone and muscle ${ }^{3,8-11}$. The TMEM16 superfamily is comprised of $\mathrm{Cl}^{-}$channels and dual function scramblases/non-selective ion channels ${ }^{4}$. Both subtypes share a common homodimeric architecture where each monomer is comprised of 10 transmembrane (TM) helices ${ }^{12-18}$ (Fig. 1A-B). In each protomer, the TM3-TM7 helices form a hydrophilic permeation pathway, or groove, that can adopt multiple conformations to allow passage of ions, lipids or to prevent movement of both substrates ${ }^{15-19}$. 
Upon activation, the TMEM16 scramblases mediate rapid lipid movement between leaflets causing the membrane asymmetry to collapse and thus initiating signaling cascades. The mechanism underlying scrambling has been investigated at the functional, computational and structural levels ${ }^{3,8,15-32}$. The consensus proposal is a 'credit-card' like mechanism ${ }^{33}$, where the lipid headgroups penetrate and traverse the open hydrophilic groove while their tails remain embedded within the hydrocarbon core of the membrane ${ }^{20,25,28}$. Within this framework, lipid scrambling is enabled by specific interactions of the permeating lipids with charged and polar groove-lining residues ${ }^{20,25,28}$. However, TMEM16 scramblases do not discriminate among lipids such as PS, PE, PG, PC and DOTAP with headgroups of different charge, structure and size $13,21,23,26$. Further, PE lipids conjugated to $5 \mathrm{kDa}$ cargoes are also efficiently scrambled ${ }^{24}$. These observations suggest that specific interactions between the groove and the scrambled lipids are not necessary. Notably, this lack of headgroup selectivity is also shared by other scramblases that lack an explicit hydrophilic groove, such as the GPCR opsin ${ }^{24,34}$ and XKR8 and $9^{6,35,36}$.

Moderate resolution structures of the fungal afTMEM16 and nhTMEM16 in nanodiscs showed these scramblases thin the membrane near the groove ${ }^{15,17}$, suggesting that membrane thinning at an open pathway might be important for lipid scrambling ${ }^{15}$. Membrane thinning was also observed near the closed pathway of mTMEM16F, leading to the proposal that scrambling can also occur outside a closed groove ${ }^{16}$. Thus, it is not clear whether an open hydrophilic groove is required for scrambling. Direct structural information on how TMEM16 scramblases interact with lipids is essential to elucidate the molecular mechanisms of lipid permeation.

Here we use cryogenic electron microscopy (cryoEM) to determine the $2.3 \AA$ resolution structure of the afTMEM16 scramblase in lipid nanodiscs. Our structure allows the direct visualization of lipids associated with the protein at the open groove and reveals that afTMEM16 
thins the membrane at the open pathway by $\sim 50 \%$. The closest point of approach of the two membrane leaflets occurs near the wide intracellular vestibule of the groove, and no lipids could be resolved inside or interacting with the extracellular portion of the pathway. Mutagenesis of groove-lining residues does not perturb function, suggesting that specific interactions of permeating lipids with groove-lining residues are not essential for scrambling. We show that in thicker membranes scrambling is inhibited, while the groove remains in an open conformation. Conversely, in thinner membranes scrambling is enhanced although the groove is closed. Thus, lipid permeation is not always enabled by an open groove or prevented by a closed pathway. Based on these findings we propose that when the groove is open, the thinned membrane and the hydrophilic nature of the pathway synergistically lower the energy barrier for lipid scrambling. When the groove is closed, scrambling can occur, but at reduced rates in bilayers with plasmamembrane like thickness. In thinner membranes, closed-groove scrambling is enhanced allowing for lipid transport in the absence of $\mathrm{Ca}^{2+}$.

\section{Results}

\section{Structural basis of lipid reorganization by the afTMEM16 scramblase}

To gain insight into how the afTMEM16 scramblase alters the organization of the membrane and interacts with the surrounding lipids we used cryo-EM to determine its structure in the $\mathrm{Ca}^{2+}$-bound conformation in nanodiscs at $2.3 \AA$ (Fig. 1, Supp. Fig. 1). Nanodiscs were comprised of a mixture of 70\% 1,2-Dioleoyl-sn-glycero-3-phosphocholine (DOPC, or 18:1 PC) and 30\% 1,2-Dioleoyl-snGlycero-3-Phosphatidylglycerol (DOPG, 18:1 PG), which we will refer to as C18 lipids. In these conditions, referred to as $\mathrm{C} 18 / \mathrm{Ca}^{2+}$, afTMEM16 is maximally active ${ }^{15}$, therefore we hypothesize this represents the active state of the scramblase. The present structure is nearly superimposable to 
the previously determined $\mathrm{Ca}^{2+}$-bound structure of afTMEM16 in 3 1-palmitoyl-2-oleoyl-snglycero-3-phosphoethanolamine (POPE): 1 1-palmitoyl-2-oleoyl-sn-glycero-3-phospho-(1'-racglycerol) POPG nanodiscs ${ }^{15}, \mathrm{C} \alpha$ rmsd $\sim 0.8 \AA$, indicating that headgroup choice and acyl-chain saturation do not influence the conformation of the protein. The significantly improved resolution of the $\mathrm{C} 18 / \mathrm{Ca}^{2+}$ map allowed us to resolve 4 water molecules in the $\mathrm{Ca}^{2+}$ binding sites which coordinate two bound ions (Supp. Fig. 2B). The presence of these water molecules brings the coordination number of bound $\mathrm{Ca}^{2+}$ ions to 7 and 8 , consistent with the high affinity of these sites ${ }^{26}$ (Supp. Fig. 2B). The map also contains non-protein densities that could be modeled as lipids associated with the protein (Fig. 1C-H, Supp. Fig. 2). To improve the quality of the density of the lipids near the pathway, we carried out symmetry expansion and additional rounds of 3D classification, which yielded one class with an additional four resolved lipids (Supp. Fig. 1E), for a total of 32 resolved lipids, 16 in each monomer (Fig. 1F-H, Supp. Fig. 2). The observed lipids define nearly continuous interfaces of the scramblase with the inner and outer membrane leaflets near the dimer interface (lipids D1-D9) and illustrate how the poses adopted by individual lipids result in the profound remodeling of the membrane induced by afTMEM16 near the lipid pathway (lipids P1-P7) (Fig. 1F-H). 

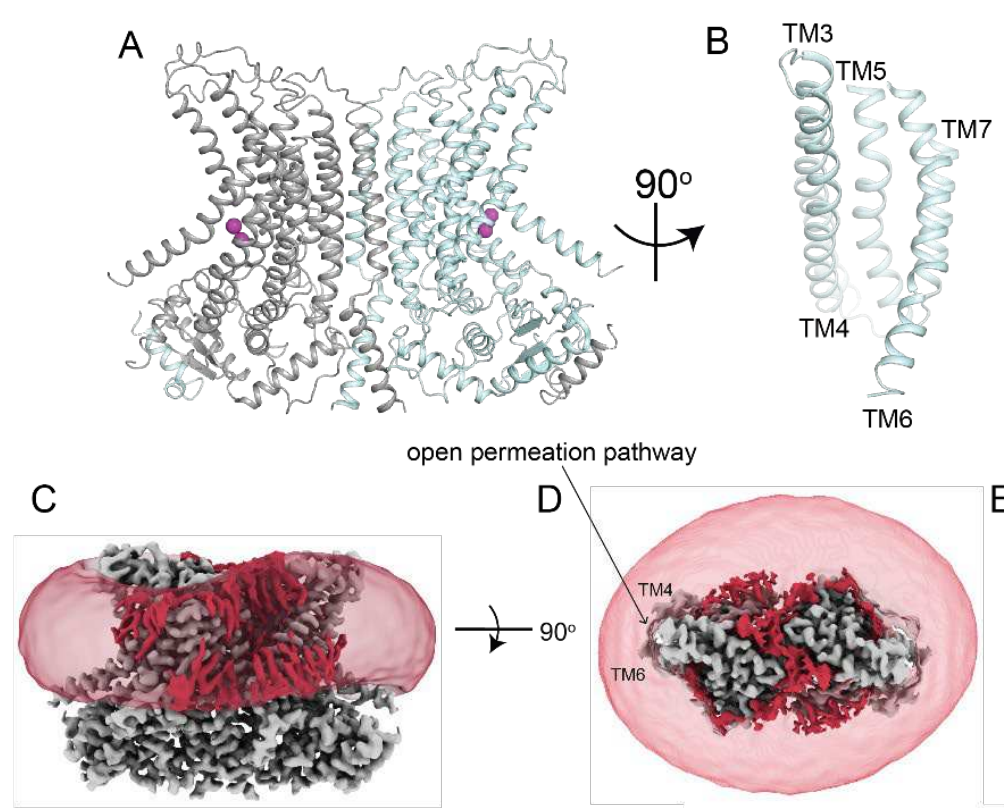

$\mathrm{E}$
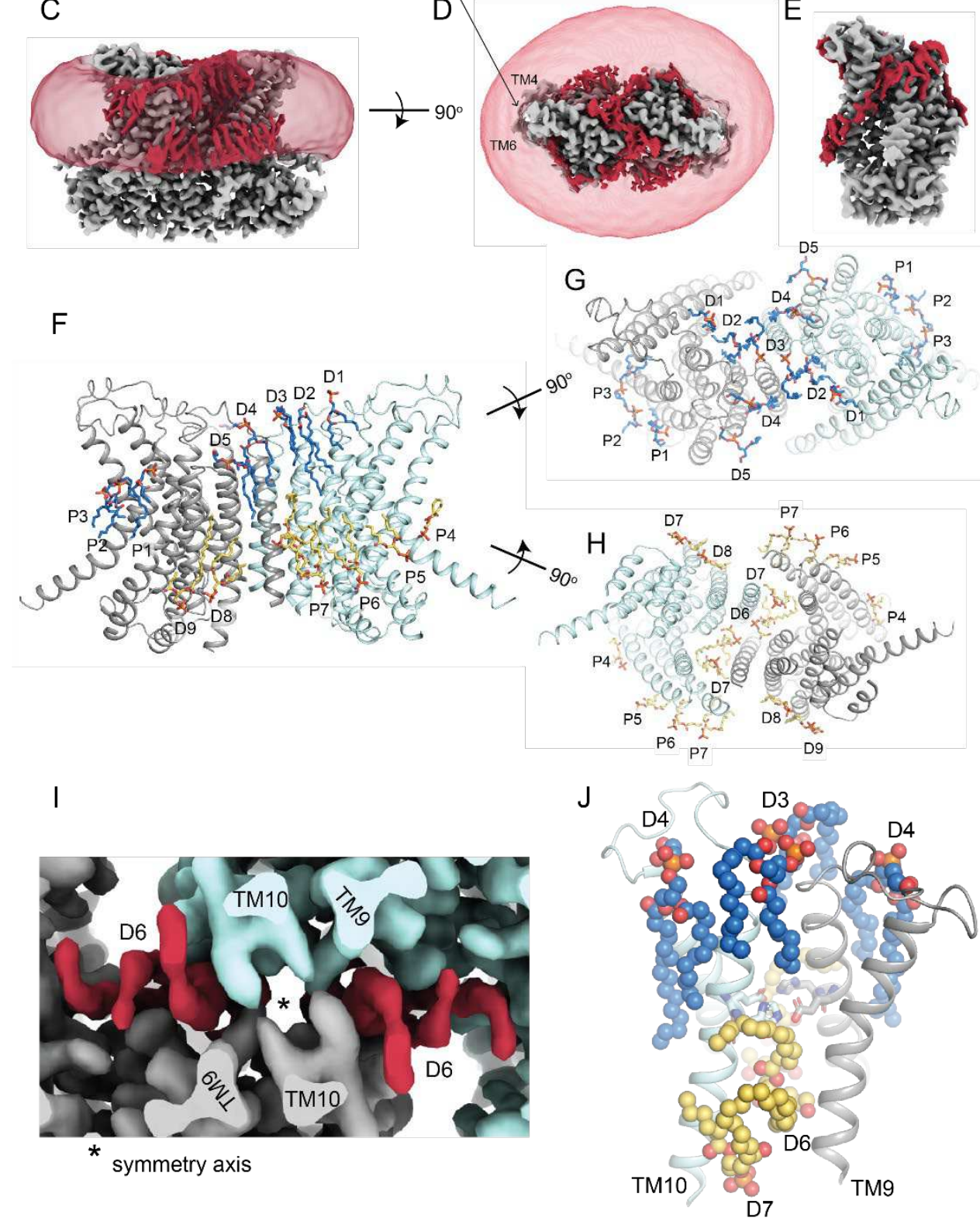

Figure 1 Lipid-protein interactions in $\mathrm{Ca}^{2+}$-bound afTMEM16. A: Structural model of afTMEM16 in $0.5 \mathrm{mM} \mathrm{Ca}^{2+}$ in $\mathrm{C} 18$ lipid nanodiscs. B: View of the open permeation pathway. CE: Unsharpened maps of the protein (grey) and associated lipids (red) viewed from the membrane 
plane (C), extracellular side (D) and close-up of the open groove (E). The map showing the density of the nanodisc membrane low-pass filtered to $10 \AA$ and shown in transparent red (C-D). F-H: Views of the afTMEM16 dimer from the plane of the membrane (F), extra- (G) and intra-cellular (H) sides with modeled lipids shown in stick representation. Lipids at the dimer interface are labeled D1-9 and those at the permeation pathway are labeled P1-7. Lipids from the inner and outer leaflets are colored in yellow and blue, respectively. The cytosolic domain of afTMEM16 was omitted for clarity. I: Close up view of the density map at the dimer interface showing the two afTMEM16 monomers (gray and cyan) and intercalated lipid tails (red). * denotes the symmetry axis. J: The dimer interface salt bridge between TM9 and 10 (in cartoon representation) is formed by E618 and H619 (in stick representation) and is shielded from the intra- and extra-cellular solutions by lipids D3, D4, D6, and D7 (in spheres and colored as in F-H).

\section{Lipids form a cap around the transmembrane dimer interface}

The transmembrane dimer interface of afTMEM16 is formed by the extracellular half of TM10 from each monomer (Fig. 1I-J, Supp. Fig. 2D-E). This minimal interface contains several hydrophobic residues and two membrane-embedded salt bridges formed by E618 and H619 of opposite subunits positioned $\sim 1 / 3$ of the way through the membrane from the extracellular leaflet (Fig. 1I-J). In the $\mathrm{C} 18 / \mathrm{Ca}^{2+}$ structure, these salt bridges appear to be isolated from the intra- and extra-cellular solutions by eight well-defined lipids (D3, D4, D6 and D7 from each subunit), four above and four below the interacting residues (Fig. 1J). On the extracellular side, the D3 lipids from opposite subunits straddle the $\mathrm{N}$ terminal region of TM10 with their heads positioned by the side chains of C607 and W608 to make direct contact above the symmetry axis (Supp. Fig. 2D). A second lipid, D4, is wedged between TM9 and TM10 with its head coordinated by polar and charged residues in the TM9-10 linker (N593, P598, T604 and R606; Supp. Fig. 2D). On the intracellular side, the heads of D6 from each subunit make contact across the symmetry axis and are wedged between the C-termini of the TM10s (Supp. Fig. 2E). They are coordinated by D571, G574 and W578 on the TM9 from one subunit and by R625, Y626 and R629 from TM10 on the

other (Supp. Fig. 2E). Additionally, the head of D7 is coordinated by Y626, S630 and K634 from TM10 of one subunit and by Q364 on TM5 and D571 on TM9 from the opposite subunit (Supp. 
Fig. 2E). The tails of these 8 lipids are accommodated in hydrophobic grooves between TM2, 9, 10 from both subunits (Fig. 1I, Supp. Fig. 2D-E). The intercalated organization of the lipid tails and helices gives rise to densely packed hydrophobic regions that shield the interacting E618 and H619 residues from water access, possibly strengthening their electrostatic interaction (Fig. 1J). These observations, together with the evolutionary conservation of the E618/H619 pair (Supp. Fig. 2C) and of the TMEM16 fold suggests these lipids might play a structural role in stabilizing the dimeric architecture of all TMEM16s.

\section{Structural basis of membrane thinning at the scrambling pathway}

The $\mathrm{C} 18 / \mathrm{Ca}^{2+}$ structure reveals how the scramblase reorients the lipids that approach the open scrambling pathway (Fig. 1F, 2A). Near the dimer interface, the planes of the outer (OL) and inner (IL) leaflets are respectively defined by lipids D1-4 and D8-9, in good agreement with the outline visualized in the low pass filtered nanodisc map (Fig. 1C). The downward slope of the OL starts at D5, a well-defined PG lipid (Fig. 1F, 2B, Fig. Supp. 2A), and progresses towards the open groove as P1 and P2 adopt distorted poses with their headgroups becoming increasingly tilted (Fig. 2A-B). The IL bends upwards and P5-P7 become increasingly tilted as their heads climb around the intracellular portions of TM3 and TM4, coordinated by the hydrophilic side chains of T341, K345 and T334 (Fig. 2A,C). Within the pathway, P3 is sandwiched between TM4 and TM6 near the constriction formed by T325 and Y432 and its headgroup points towards the extracellular side such that it is contiguous to other OL lipids (Fig. 2A). The distance between the phosphate atoms of the headgroups of $\mathrm{P} 3$ and $\mathrm{P} 4$ in the $\mathrm{OL}$ and IL is $<22 \AA$ (Fig. 2A), showing that the hydrocarbon core of the membrane is thinned by $\sim 50 \%$ at the open pathway. A similar thinning is seen in the low-pass filtered nanodiscs map near the pathway (Fig. 1C-D). 


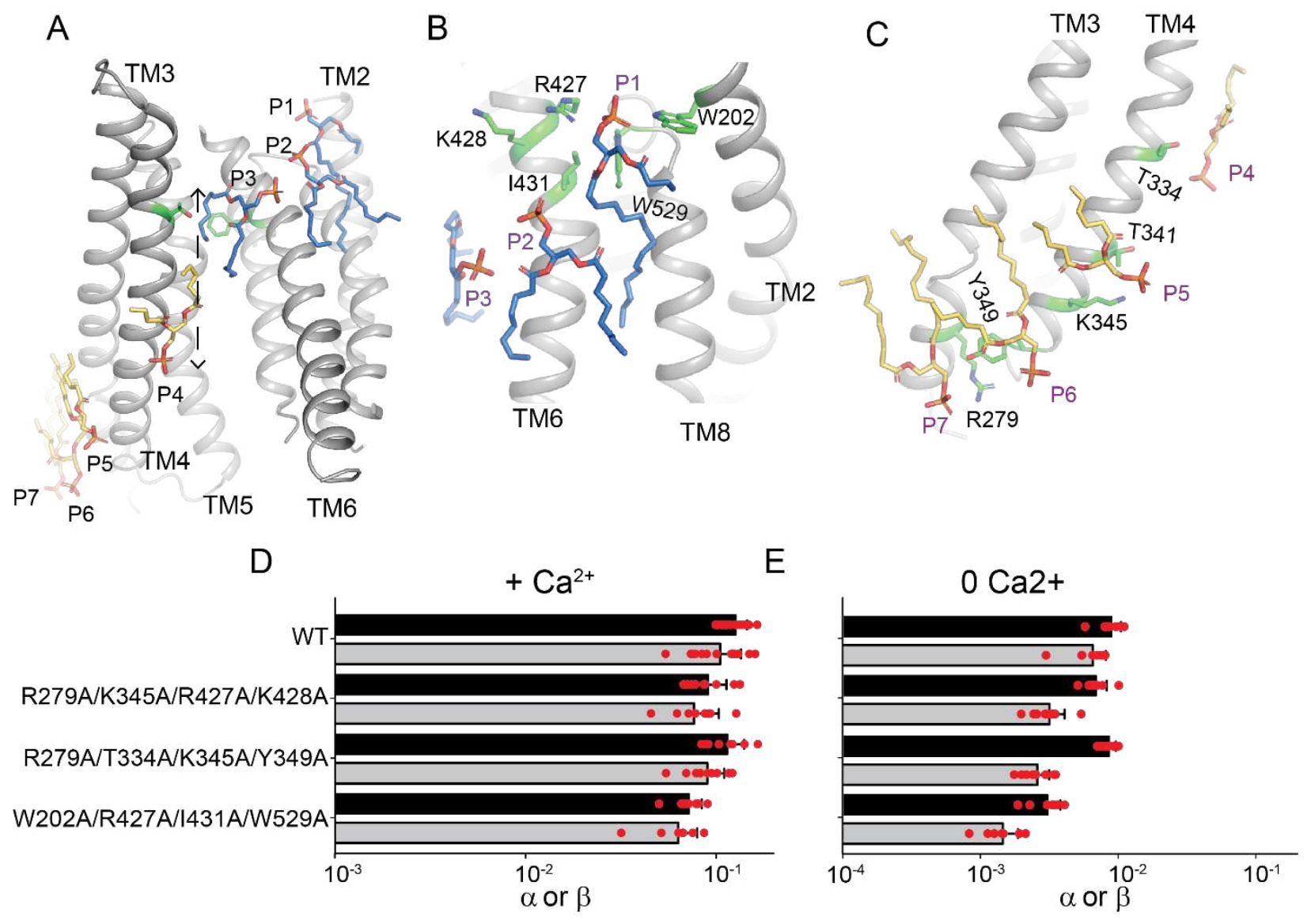

Figure 2. Coordination of lipids outside the permeation pathway. A: View of the seven pathway lipids (in sticks, colored as in Fig. 1F). T325 and Y423 are shown as green sticks. Dashed arrow indicates the distance between the phosphate atoms of the last lipid from the inner (P4) and outer (P3) leaflets. B-C: Coordination of P1-P2 (B) and of P4-P7 (C). Side chains are shown in green sticks. D-E: forward $(\alpha)$ and reverse $(\beta)$ scrambling rate constants for indicated quadruple mutants of residues coordinating lipids outside the pathway (P1-2 and P4-7). Bars are average values for $\alpha$ (black) and $\beta$ (grey), error bars are S. Dev., and red circles are values from individual repeats.

\section{Lipids outside the open pathway define the distorted membrane interface}

The identification of sites where lipids bind at or near the open groove raises the possibility that scrambling could occur via a 'conveyor belt' mechanism, where lipids translocate between leaflets by moving from site to site. Alternatively, the observed lipids could define the protein-membrane boundary but not necessarily be translocated, with the possible exception of P3 within the pathway 
(Fig. 2A). To distinguish between these hypotheses, we investigated how mutating residues coordinating the headgroups of P1-2 and P4-6 impacts scrambling. We found that mutations aimed at disrupting the headgroup interactions of P1-P2 (W202A/R427A/I431A/W529A), P4-P5-P6 (R279A/T334A/K345A/Y349A) or P2-P5-P6 (R279A/K345A/R427A/K428A) have minimal functional effects (Fig. 2D-E, Supp. Fig. 3). This suggests that these lipid association sites are not obligatory on the path taken by scrambled lipids. Rather, other factors, such as tail interactions with interhelical grooves, contribute to their association with afTMEM16 (Supp. Fig 2F-G) and stabilize the distorted membrane-protein interface that results in thinning at the pathway.

\section{Scrambling does not require specific interactions with extracellular groove-lining residues}

One unexpected feature of our structure is that the extracellular vestibule of the groove does not directly interact with the membrane and no lipids could be resolved (Fig. 1C-D), as they traverse the groove below this region (Fig. 2A). We mutated side chains lining the extracellular vestibule or the central constriction of the groove and assessed their impact on scrambling. Single or multiple simultaneous alanine substitutions of I298, F302, E305 and E310 on TM3, of K317, Y319, F322, T325 and I332 on TM4, of T373 and S374 on TM5 and of R425, K428, Q429, Y432 and F433 on TM6 have no effects on lipid scrambling (Fig. 3, Supp. Fig. 3). Thus, scrambling does not entail specific interactions of lipids with residues lining the extracellular vestibule or the central constriction of the groove.

In contrast, the wide intracellular vestibule is embedded in the nanodisc membrane, and the resolved P3 and P4 lipids at the open pathway have opposite orientations (Fig. 2A). This suggests lipid headgroups only need to traverse the wide intracellular vestibule of the pathway, below the constriction formed by T325 and Y432 (Fig. 2A). The pronounced membrane thinning 
at the pathway lowers the energy barrier for transbilayer lipid movement and the hydrophilic environment of the open groove allows water access to this thinned membrane region, synergistically lower the energy barrier for scrambling. Scrambling by afTMEM16 and hTMEM16K is modulated by lipid acyl chain length ${ }^{15,23}$, supporting the idea that membrane thinning is critical for scrambling. Our proposal predicts that this modulation should reflect whether the scramblase can sufficiently thin these membranes, rather than arise from lipiddependent changes in the conformation of the groove.
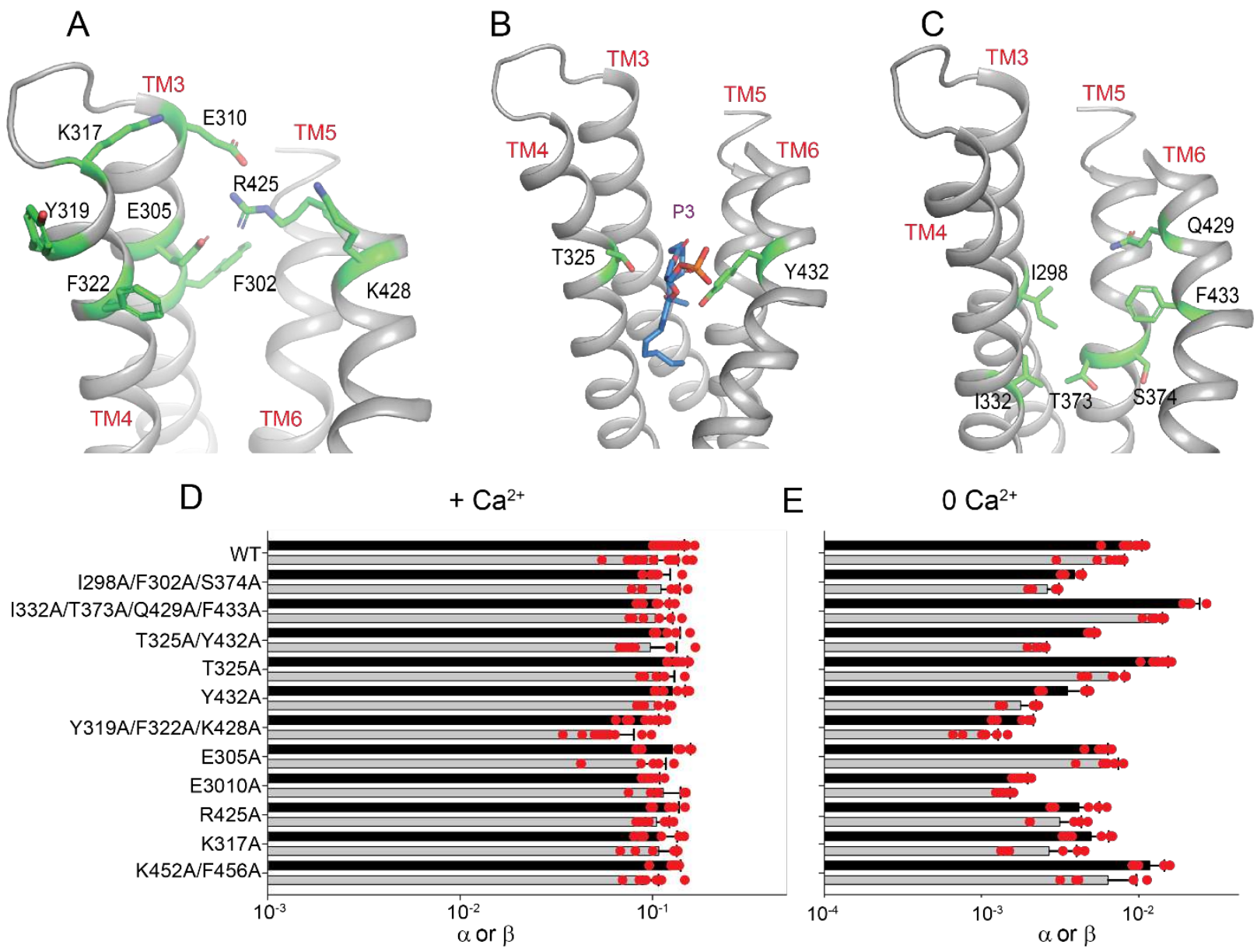

Figure 3. Functional role of groove-lining residues in lipid scrambling. A-C: Residues lining the extracellular vestibule (A), coordinating P3 (B) and lining the central constriction (C) are shown as green sticks. D-E: forward $(\alpha)$ and reverse $(\beta)$ scrambling rate constants of single and multiple alanine substitutions at the indicated positions. Bars are average values for $\alpha$ (black) and $\beta$ (grey), error bars are $S$. Dev., and red circles are values from individual repeats. 


\section{Regulation of lipid scrambling by membrane thickness}

We measured how systematic variation of lipid acyl chain length affects lipid scrambling by afTMEM16. We kept the lipid headgroup composition at a constant ratio of 7 PC: 3 PG and used acyl chains with a single unsaturation and 16-22 carbons, C16-C22 lipids (Table 1). Liposomes formed from this mix of 14:1 lipids were not stable in our scrambling assay (Supp Fig. 4C), therefore we generated thinner membranes using a mixture comprised of 50\% 1,2-dimyristoyl-snglycero-3-phosphocholine (DMPC) and 1,2-dimyristoyl-sn-glycero-3-phospho-(1'-rac-glycerol) (DMPG) in a 7:3 ratio and 50\% of POPC and POPG in a 7:3 ratio ${ }^{23}$; we will refer to this mix as C14 (Table 1). Atomic force microscopy (AFM) measurements show that membrane thickness varies between $\sim 3.2 \mathrm{~nm}$ and $\sim 4.1 \mathrm{~nm}$, with near-linear scaling with acyl chain length (Table 1 , Supp. Fig. 4A-B).

In the presence of saturating $0.5 \mathrm{mM} \mathrm{Ca}^{2+}$ the scrambling rate constants do not depend on membrane thickness between $\sim 3.2 \mathrm{~nm}$ (C14 lipids) and $\sim 3.9 \mathrm{~nm}$ (C20 lipids) (Fig. 4A). In contrast, scrambling is nearly completely inhibited in C22 lipids (Fig. 4A) ${ }^{15}$. Thus, in saturating $\mathrm{Ca}^{2+}$ there is chain length selectivity with a threshold for activity below membrane thickness of $\sim 4.1 \mathrm{~nm}$. In contrast, in $0 \mathrm{Ca}^{2+}$ scrambling displays a nearly exponential inverse dependence on membrane thickness (Fig. 4B). Remarkably, in C14 lipid membranes scrambling by afTMEM16 is nearly $\mathrm{Ca}^{2+}$-independent, with rate constants only $\sim 3$-fold lower in $0 \mathrm{Ca}^{2+}$ compared to the $\sim 20$-fold reduction seen in C18 membranes (Fig. 4A-B, Supp. Fig. 4). To test whether the long C22 acyl chains inhibit scrambling in saturating $\mathrm{Ca}^{2+}$ by occluding the pathway ${ }^{37}$ we measured scrambling in membranes formed by 70\% C22 lipids and 30\% C18 lipids, which are $\sim 4 \mathrm{~nm}$ thick (Table 1). In saturating $\mathrm{Ca}^{2+}$ scrambling activity is similar to that seen in $100 \% \mathrm{C} 18$ lipids (Fig. 4A), while in $0 \mathrm{Ca}^{2+}$ there is a $\sim 17$-fold reduction, consistent with the reduction expected for membranes of 
this thickness (Fig. 4B). This behavior does not depend on whether the mixed chain lengths were segregated by headgroup. Thus, the tails of C22 lipids are not 'blockers' of the afTMEM16 permeation pathway. These results suggest that in $0 \mathrm{Ca}^{2+}$ scrambling rates are proportional to the energetic cost of lipid headgroups crossing the hydrophobic core of the membrane, while in the presence of $\mathrm{Ca}^{2+}$ other factors contribute to scrambling.

\section{$\mathrm{Ca}^{2+}$-bound afTMEM16 has an open groove in $\mathrm{C} 22$ membranes}

To determine whether the $\mathrm{C} 22$ lipids inhibit scrambling by inducing groove closure we determined the cryo-EM structure of nanodisc-reconstituted afTMEM16 in the presence of saturating $\mathrm{Ca}^{2+}$ to $2.7 \AA$ (Supp. Fig. 5A-G). Despite a $\sim 500$-fold reduction in scrambling activity the groove remains open in a conformation nearly identical to that seen in C18 lipids, C $\alpha$ r.m.s.d. $\sim 0.35 \AA$ (Fig. 4D). Importantly, neither the $\mathrm{C} 18 / \mathrm{Ca}^{2+}$ nor the $\mathrm{C} 22 / \mathrm{Ca}^{2+}$ datasets display structural heterogeneity as no additional classes could be identified using multiple rounds of iterative 3D classifications on afTMEM16 dimers and monomers using different classification parameters and software (see Methods, Supp. Fig. 1,5, 7, 8, 10). Further, The $\mathrm{C} 22 / \mathrm{Ca}^{2+}$ structure of afTMEM16 in the larger MSP2N2 nanodiscs at $3.5 \AA$ resolution (Supp. Fig. $5 \mathrm{H}-\mathrm{N}$ ) shows an open permeation pathway in all 3D reconstructions, with $\mathrm{C} \alpha$ r.m.s.d. $\sim 0.5 \AA$ to $\mathrm{C} 18 / \mathrm{Ca}^{2+}$ and $\sim 0.4 \AA$ to $\mathrm{C} 22 / \mathrm{Ca}^{2+}{ }_{\mathrm{MSP}}$ (E3 3 (Fig. 4D). Thus, in afTMEM16 an open groove is not sufficient to enable lipid scrambling and nanodisc size does not influence the conformation.

In the $\mathrm{C} 22 / \mathrm{Ca}^{2+}$ maps we resolved several of the lipids near the dimer interface corresponding to D2, D3, D6, and D7 (in MSP1E3 map) and to D2 and D6 (in MSP2N2 map) seen in the $\mathrm{C} 18 / \mathrm{Ca}^{2+}$ map (Supp. Fig. 6). In the MSP1E3 map we detected strong density for P6 and P7, located near the intracellular loop connecting TM3 and TM4 (Supp. Fig. 6). However, despite 
the high resolution of the $\mathrm{C} 22 / \mathrm{Ca}^{2+}$ MSP1E3 map, we detect only weak signals for lipids associated with the pathway-delimiting helices TM4 and TM6. This suggests that the interactions of C22 lipids with the pathway helices are weaker than those of C18 lipids, likely reflecting the higher energy cost associated with distorting these longer acyl chain lipids.

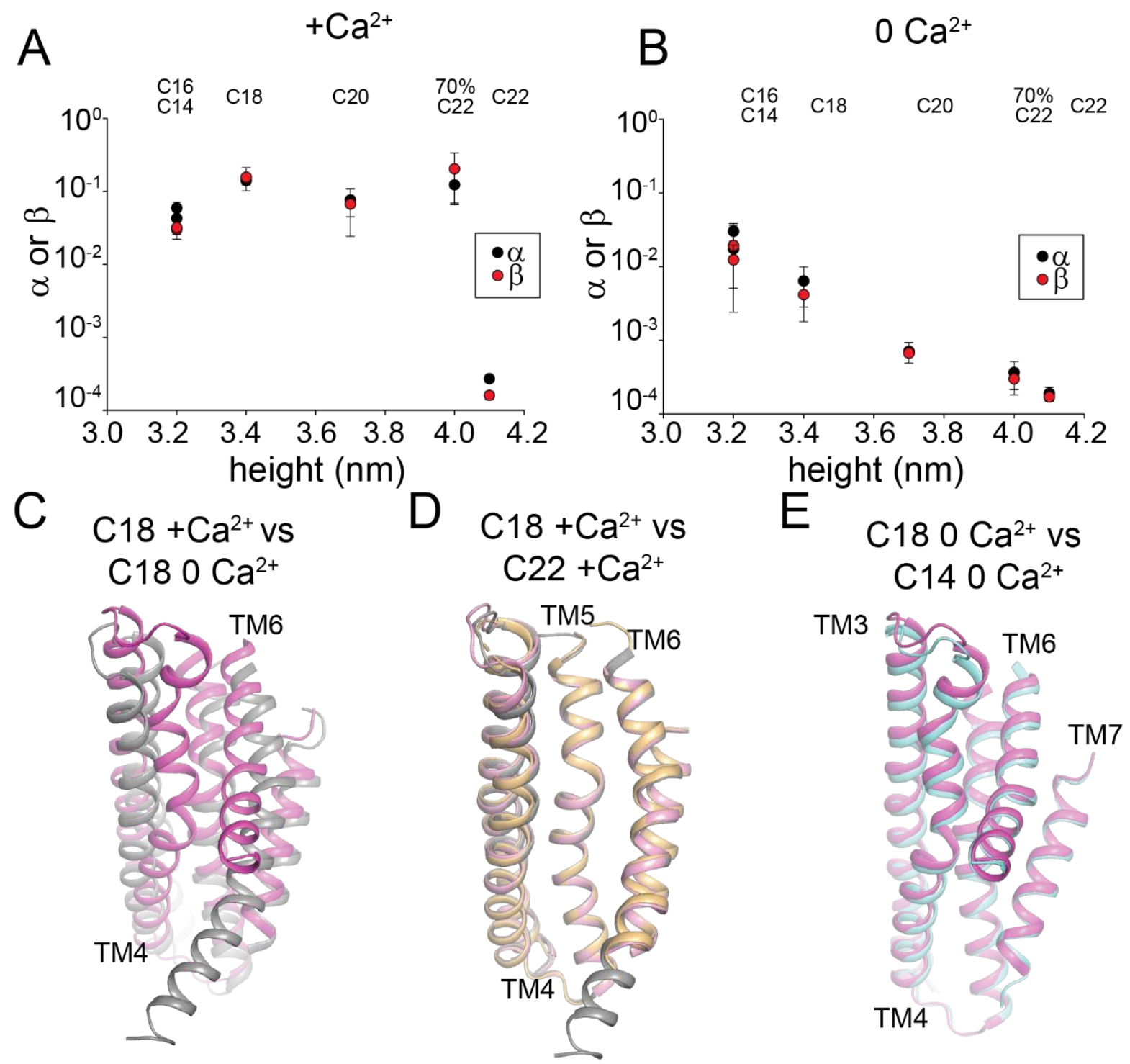

Figure 4. Functional and structural regulation of lipid scrambling by membrane thickness. A-B: Forward ( $\alpha$, black circles) and reverse ( $\beta$, red circles) scrambling rate constants as a function of membrane thickness in the presence of $0.5 \mathrm{mM}(\mathrm{A})$ or $0 \mathrm{Ca}^{2+}(\mathrm{B})$. Values are the mean and error bars represent standard deviation. Corresponding lipid compositions are noted above. C-E: Alignment of the permeation pathway of afTMEM16 in (C) C18 nanodiscs in $0.5 \mathrm{mM}$ (grey) or 0 
$\mathrm{Ca}^{2+}$ (pink), (D) in $0.5 \mathrm{mM} \mathrm{Ca}^{2+}$ and $\mathrm{C} 18$ (grey) or C22 MSP1E3 nanodiscs (light pink) or C22 MSP2N2 nanodiscs (orange), (E) in $0 \mathrm{Ca}^{2+}$ in $\mathrm{C} 18$ (red) and $\mathrm{C} 14$ nanodiscs (cyan).

\section{Scrambling in $0 \mathrm{Ca}^{2+}$ does not require groove opening}

These finding that an open groove is not sufficient to allow lipid movement raises the question of whether a closed groove prevents lipid scrambling entirely. Many proteins that scramble lipids lack explicit membrane-exposed hydrophilic grooves ${ }^{34-36,38}$ and most purified TMEM16's scramble lipids in $0 \mathrm{Ca}^{2+}$ when the groove is predominantly closed (Fig. 4B) ${ }^{13,23,26}$. This basal activity could reflect transient openings of the pathway, however an open groove $\mathrm{Ca}^{2+}$-free conformation has not been observed in a membrane environment ${ }^{15-18}$. Alternatively, these scramblases could thin the membrane enough to enable slow lipid scrambling outside of a closed groove, as proposed for the mammalian TMEM16F ${ }^{16}$.

To elucidate the structural bases of scrambling in the absence of $\mathrm{Ca}^{2+}$, we determined the $3.1 \AA$ resolution structure of afTMEM16 in $\mathrm{C} 18$ lipids in $0 \mathrm{Ca}^{2+}$ (Supp. Fig. 7). Extensive classification of afTMEM16 dimers and of symmetry-expanded monomers (see Methods) revealed only reconstructions corresponding to a closed groove conformation (Fig 4C, Supp. Fig. 7). However, in C18 lipids the basal scrambling activity of afTMEM16 is modest, $\sim 4.5 \%$ of that in saturating $\mathrm{Ca}^{2+}$ (Fig. 4A-B, Supp. Fig. 4), suggesting that the fraction of particles that could adopt a $\mathrm{Ca}^{2+}$-free open groove conformation could be too small to be detected. In contrast, in $\mathrm{C} 14$ lipids scrambling in $0 \mathrm{Ca}^{2+}$ is only $\sim 3$-fold slower than in saturating $\mathrm{Ca}^{2+}$ (Fig. 4A-B, Supp. Fig 4) so that a significant portion of the particles should adopt a $\mathrm{Ca}^{2+}$-free open-groove conformation. Analysis of a C14/0 $\mathrm{Ca}^{2+}$ afTMEM16 dataset yields only classes with a closed groove (Fig. 4E), $\mathrm{C} \alpha$ r.m.s.d. $\sim 0.9 \AA$ to $\mathrm{C} 18 / 0 \mathrm{Ca}^{2+}$, the highest of which reached $3.3 \AA$ average resolution (Supp. Fig. 8). Thus, in $0 \mathrm{Ca}^{2+}$ there is a $\sim 30$-fold increase in scrambling between $\mathrm{C} 14$ and $\mathrm{C} 18$ lipid 
membranes that is not accompanied by an increase in the probability of opening of the groove. This suggests that the basal, $\mathrm{Ca}^{2+}$ independent activity is due to closed-groove scrambling.

This hypothesis is further supported by the analysis of the D511A/E514A mutant of afTMEM16 that disrupts the $\mathrm{Ca}^{2+}$-binding site. This mutation impairs TMEM16 activity by preventing opening of the pathway ${ }^{8,26,39,40}$ and scrambles lipids in a $\mathrm{Ca}^{2+}$-independent manner at rates comparable to those of the WT protein in $0 \mathrm{Ca}^{2+}{ }^{26}$. Scrambling by D511A/E514A afTMEM16 is modulated by membrane thickness like the WT protein in $0 \mathrm{Ca}^{2+}$ (Fig. 5A, Supp. Fig. 9), so that in C14 membranes its activity is $\sim 30 \%$ of that of the WT protein in C18 lipids and saturating $\mathrm{Ca}^{2+}$. To test whether the D511A/E514A afTMEM16 adopts an open-pathway conformation in conditions of high activity, we determined its structure in C14 nanodiscs with 0.5 $\mathrm{mM} \mathrm{Ca}^{2+}$ to $3.1 \AA$ resolution (Supp. Fig. 10). As expected, despite the presence of $0.5 \mathrm{mM} \mathrm{Ca}^{2+}$, the protein adopts the same conformation as in the WT apo structure and there is no density in the $\mathrm{Ca}^{2+}$ binding site. In all reconstructions the permeation pathway is closed, with $\mathrm{C} \alpha$ r.m.s.d. $\sim 1.1 \AA$ to $\mathrm{C} 18 / 0 \mathrm{Ca}^{2+}$ and $\sim 0.4 \AA$ to $\mathrm{C} 14 / 0 \mathrm{Ca}^{2+}$ (Supp. Fig. 10), indicating that increased scrambling is not accompanied by higher open probability of the groove. Together, our results suggest that scrambling of afTMEM16 in $0 \mathrm{Ca}^{2+}$ occurs outside of a closed groove. Calcium-independent openings of the lipid permeation pathway, if they occur, are transient and cannot account for the observed increase in activity. Thus, an open groove is not necessary for lipid scrambling.

In the three datasets for apo afTMEM16 $\left(\mathrm{C} 18 / 0 \mathrm{Ca}^{2+}, \mathrm{C} 14 / 0 \mathrm{Ca}^{2+}\right.$ and DA/EA in $0.5 \mathrm{mM}$ $\mathrm{Ca}^{2+}$ ) we could resolve 4-9 lipids per monomer, all localized near the dimer interface in positions closely resembling those seen in $\mathrm{C} 18 / \mathrm{Ca}^{2+}$ structure (Supp. Fig. 11), supporting the notion that these lipids interact strongly with the protein. No lipids could be resolved near the closed pathway 
in these structures. The average resolution of these datasets is lower than that of the two $\mathrm{Ca}^{2+}-$ bound structures, preventing us from drawing mechanistic inferences from this observation.
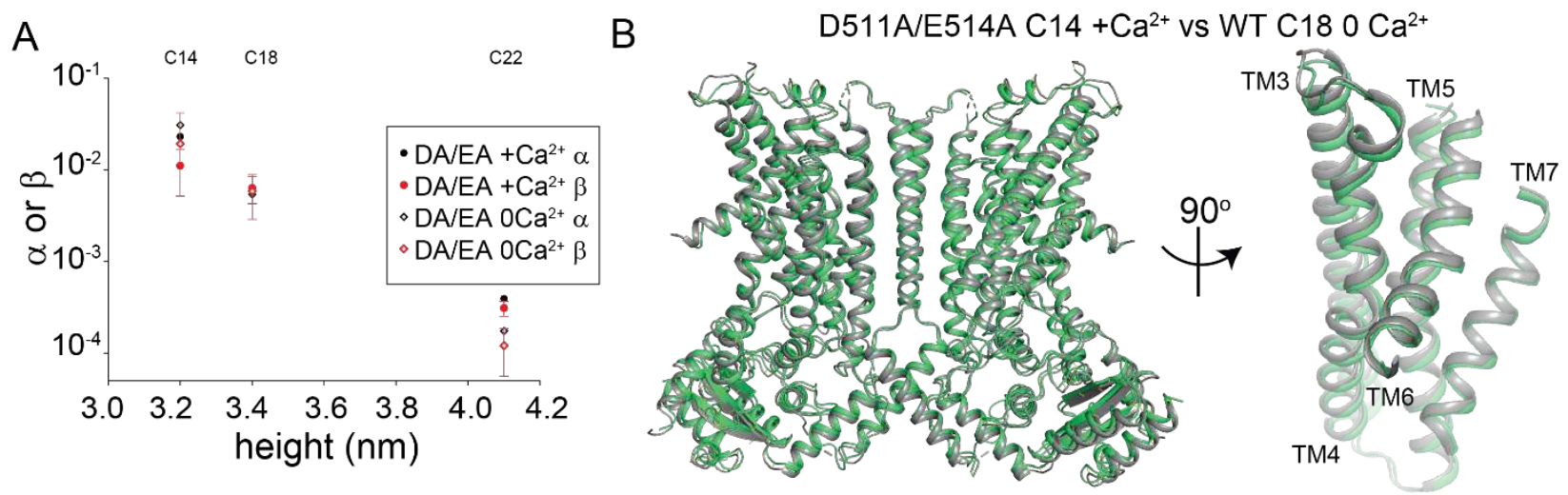

Figure 5. Functional and structural characterization of afTMEM16 D511A/E514A. A-B: Forward ( $\alpha$, black circles) and reverse ( $\beta$, red circles) scrambling rate constants of D511A/E514A afTMEM16 in $0.5 \mathrm{mM}$ (filled symbols) or $0 \mathrm{Ca}^{2+}$ (empty symbols). Values are the mean and error bars represent standard deviation. Corresponding lipid compositions are noted above. B: Alignment of afTMME16 D511A/E514A in the presence of $\mathrm{Ca}^{2+}$ (green) in C14 lipids with wildtype afTMEM16 in $0 \mathrm{Ca}^{2+}$ in $\mathrm{C} 18$ lipids (grey) with close up view of the permeation pathway.

\section{Scrambling activity correlates with membrane thinning at the pathway}

Our proposal that afTMEM16 enables scrambling by thinning the membrane at the permeation pathway predicts there should be a correlation between thinning and function. Although a quantitative evaluation of thinning is precluded by the different resolutions of the maps, a qualitative analysis of the nanodisc density maps supports this notion (Fig. 6). Far from the protein, membrane thickness of $\mathrm{C} 14, \mathrm{C} 18$ and $\mathrm{C} 22$ nanodiscs is comparable to that determined by AFM (Table 1). Near the open groove, in the $\mathrm{C} 18 / \mathrm{Ca}^{2+}$ map the membrane appears significantly thinned (Fig. 6B), closely tracking the position of individual lipids (Fig. 2A). Thinning is reduced near the open pathway of the $\mathrm{C} 22 / \mathrm{Ca}^{2+}$ map (Fig. $6 \mathrm{~A}$ ) and near the closed pathway of the $\mathrm{C} 18 / 0 \mathrm{Ca}^{2+}$ map (Fig. 6C), consistent with the reduced scrambling activity (Fig. 4A-B). In the $\mathrm{C} 14 / 0 \mathrm{Ca}^{2+}$ map, thickness at the closed pathway qualitatively approaches that at the open pathway of the $\mathrm{C} 18 / \mathrm{Ca}^{2+}$ 
map (Fig. 6D), consistent with enhanced scrambling (Fig. 4B). These qualitative observations suggest there is a direct correlation between the thickness of the membrane near the pathway and scrambling activity. This supports the idea that in C22 membranes scrambling could be inhibited because of the reduced thinning despite an open groove, and that the closed groove conformation of afTMEM16 is scrambling competent because it thins the membrane enough to enable lipid flipping.

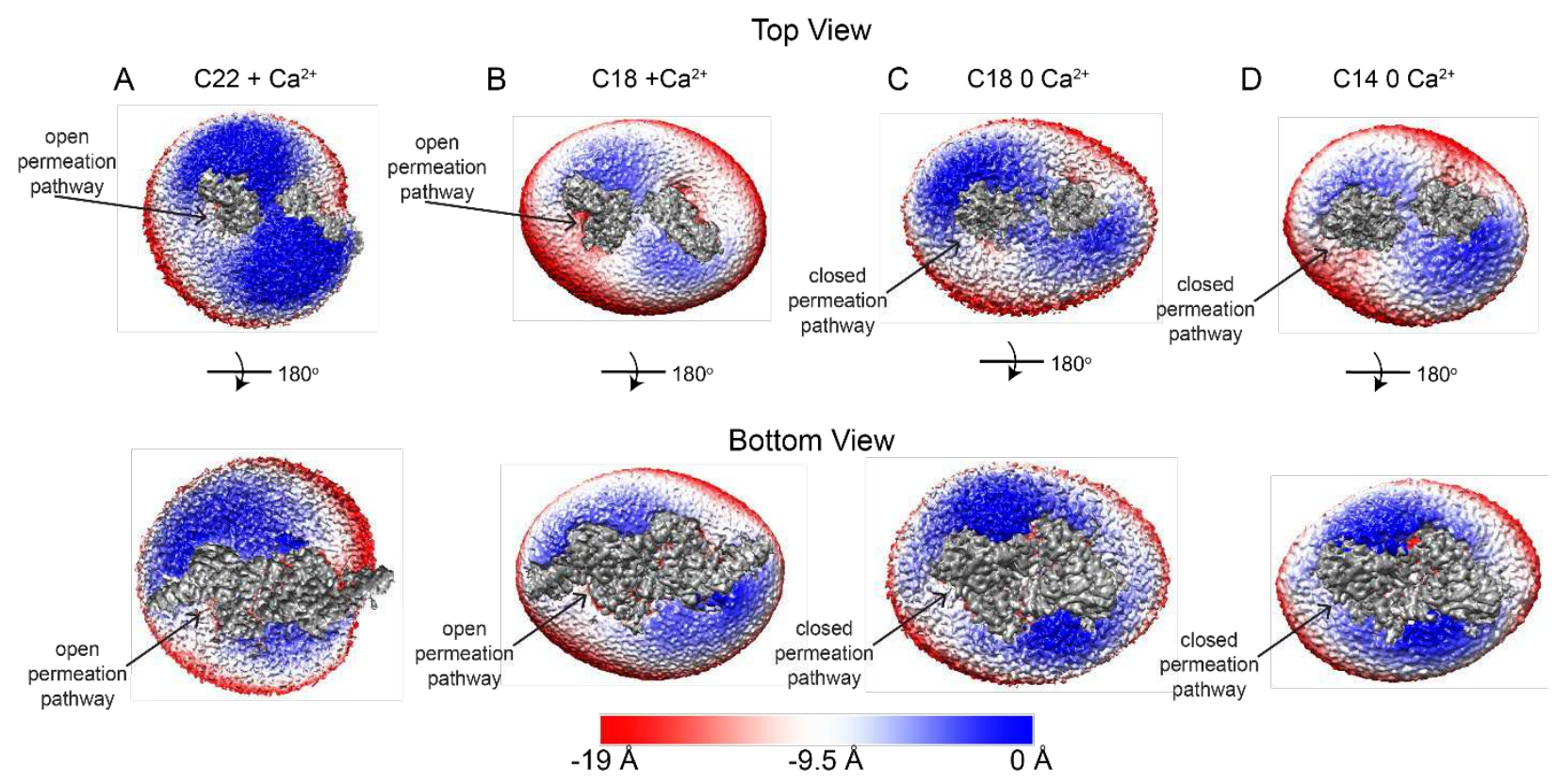

Figure 6. Membrane thinning at the afTMEM16 pathway as a function of acyl chain length. A-D: Views of the density maps for afTEM16 in C22/ $\mathrm{Ca}^{2+}(\mathrm{A}), \mathrm{C} 18 / \mathrm{Ca}^{2+}(\mathrm{B}), \mathrm{C} 18 / 0 \mathrm{Ca}^{2+}(\mathrm{C})$ and $\mathrm{C} 14 / 0 \mathrm{Ca}^{2+}(\mathrm{D})$ from the extracellular (top panels) and intracellular (bottom panel) side. The $\mathrm{C} 1$ final unsharpened maps containing nanodisc densities were aligned, resampled on the same grid, and colored according to the $\mathrm{Z}$ coordinate using UCSF Chimera. The density corresponding to the protein is segmented and shown in gray. Nanodisc densities are colored by displacement along the $\mathrm{Z}$ axis and the $0 \AA$ reference height is the same for all structures in each view. Negative values represent membrane thinning relative to the overall nanodisc. The position of the permeation pathway is denoted with arrows.

\section{Discussion}

Activation of scramblases catalyzes the rapid movement of phospholipids between membrane leaflets and results in the externalization of charged and polar lipids that trigger a variety of 
fundamental physiological processes ${ }^{1,2,4}$. The current consensus is that TMEM16 scramblases mediate lipid transport via a credit-card like mechanism ${ }^{33}$, with the headgroups forming specific interactions with polar and charged residues lining the full length of the hydrophilic groove 20,23,25,28. This predicts that scramblases should discriminate among lipids based on their headgroups but not their tails, and that mutations of groove-lining residues should affect lipid scrambling. Notably, neither scramblases like the TMEM16's ${ }^{13,18,21,24,26}$ or the Xkr's ${ }^{6,41}$ nor GPCR's moonlighting as scramblases ${ }^{34}$, show selectivity among lipids with different headgroups. Further, both the Xkr's and GPCR's lack explicit hydrophilic grooves ${ }^{34-36}$, bringing the structural requisites of the credit card mechanism into question. However, the modes of lipid-protein interactions of TMEM16 scramblases had not been structurally resolved ${ }^{15-18}$.

Here, we combined structural and functional experiments to investigate the mechanism of lipid scrambling by the TMEM16's. The $2.3 \AA$ structure of afTMEM16 reconstituted in C18 nanodiscs shows how individual lipids interact with the scramblase to define the thinned and distorted profile of the membrane near the open pathway (Fig. 1C-H). Lipids mainly localize to the periphery of the groove, interacting with the intracellular portions of TM3-4 and with the extracellular portions of TM6-7. The position of the last lipids from the intra- and extra-cellular leaflets suggests that headgroup flipping occurs in the intracellular vestibule. No density for lipids was visible near the extracellular vestibule (Fig. 1,2A) and mutations of residues lining this narrow constriction or the groove interior have no functional effects (Fig. 2, 3). Reconstituting afTMEM16 in membranes formed from lipids with longer acyl chains dramatically inhibits scrambling although the groove remains open (Fig. 4). Conversely, reconstitution in thinner membranes facilitates scrambling even when the groove is closed (Fig. 4-5). 
Together, these results have three important implications; first, lipid scrambling does not entail specific interactions with the groove's hydrophilic interior or its extracellular vestibule. Second, acyl chains rather than headgroups are key determinants of scrambling activity (Fig. 4AB). Third, an open groove is neither sufficient nor necessary for scrambling (Fig. 4, 5). These findings are inconsistent with a credit-card mechanism. Rather, we propose that lipid scrambling is primarily determined by the ability of afTMEM16 to thin the membrane near the pathway, so that lipids only interact with the surface of the groove without penetrating deep within its narrow and hydrophilic interior (Fig. 7). The membrane-thinning mechanism readily explains evolutionarily conserved properties of TMEM16 scramblases that are difficult to reconcile with the credit card mechanism, such as the lack of discrimination based on headgroup size, chemistry or charge ${ }^{13,18,21,23,26,42}$ and scrambling of lipids conjugated to large cargoes ${ }^{24}$. Thus, we propose this mechanism applies to other TMEM16's.

Two lines of evidence support the credit card hypothesis: mutating groove-lining residues impairs lipid scrambling by nhTMEM16 and TMEM16F ${ }^{25,28}$ and MD simulations show lipid headgroups penetrating and traversing the whole length of the groove ${ }^{20,23,25,28,43,44}$. Strikingly we find that mutating similar residues in afTMEM16 does not impair scrambling (Fig. 2,3). This contradiction could be explained if the mutants impair scrambling by favoring groove closure rather than by impairing lipid movement through an open groove. In afTMEM16 only the $\mathrm{Ca}^{2+}-$ bound open conformation has been observed ${ }^{15}$ (Fig. 1, 4). In contrast, $\mathrm{Ca}^{2+}$-bound nhTMEM16 adopts both open and closed groove conformations ${ }^{17}$ and in mTMEM16F only the $\mathrm{Ca}^{2+}$-bound closed groove conformation has been observed ${ }^{16,18}$, suggesting in these homologues the $\mathrm{Ca}^{2+}$ bound open conformation is less stable than in afTMEM16. Further, several scramblingincompetent nhTMEM16 mutants retain measurable channel activity ${ }^{25}$, suggesting stabilization 
of an ion channel-like groove conformation ${ }^{19}$. Discrepancies with molecular dynamics simulations could be due to incomplete relaxation of the membrane during the equilibration, especially if the chosen initial conditions for the protein-lipid arrangements are far from equilibrium. Indeed, recent work suggests extended equilibration protocols are needed to capture the full extent of membrane deformations induced by some proteins ${ }^{45}$. Further, our structures show several lipids with tails tightly intercalated with TM helices at the dimer cavity (Fig. 1) that might affect the dynamic rearrangements of afTMEM16 in MD simulations. It will be interesting to see how incorporating new information on the modes of lipid interactions with the afTMEM16 scramblase affects these results.

The implications of our proposed membrane-thinning mechanism for scrambling (Fig. 7) can be appreciated if we make the simplifying assumptions that (i) the energy barrier for lipid scrambling is due to the polar head and (ii) that the head can be modeled with a sphere of radius $r$ and charge $q$, then the energy barrier for scrambling, $\Delta G_{\text {scramb }}$ would be given by ${ }^{46}$

$$
\Delta G_{\text {scramb }}=\frac{q^{2}}{2 \varepsilon_{m} r}-\frac{q^{2}}{\varepsilon_{m} L} \ln \left(\frac{2 \varepsilon_{w}}{\varepsilon_{m}+\varepsilon_{w}}\right)
$$

Where $\varepsilon_{\mathrm{m}, \mathrm{w}}$ are the dielectric constants of the membrane and of water and $\mathrm{L}$ is the thickness of the membrane. Membrane thinning decreases $\Delta G_{\text {scramb }}$ as $\mathrm{L}$ is reduced and the dielectric constant $\varepsilon_{\mathrm{m}}$ is increased because of higher water access to the hydrocarbon core of the membrane ${ }^{47}$. When the pathway is open scrambling is fast because thinning is pronounced, and the hydrophilic interior of the open groove further decreases $\varepsilon_{\mathrm{m}}$ (Fig. 7A). In thicker or more rigid membranes (Fig. 4) ${ }^{15}$, scrambling is impaired because their deformation cost is higher thus preventing lipids to reach positions conducive to scrambling (Fig. 7B). When the groove is closed membrane thinning is diminished, but not absent, which allows for slow scrambling activity (Fig. 7C), that is enhanced in membranes formed from shorter chain length lipids (Fig. 7D). Notably, the proposed membrane- 
thinning mechanism could naturally explain how proteins lacking hydrophilic grooves, such as GPCR's and Xkr's, can scramble lipids and share functional properties with the structurally unrelated TMEM16's ${ }^{24,34-36,38,48}$.
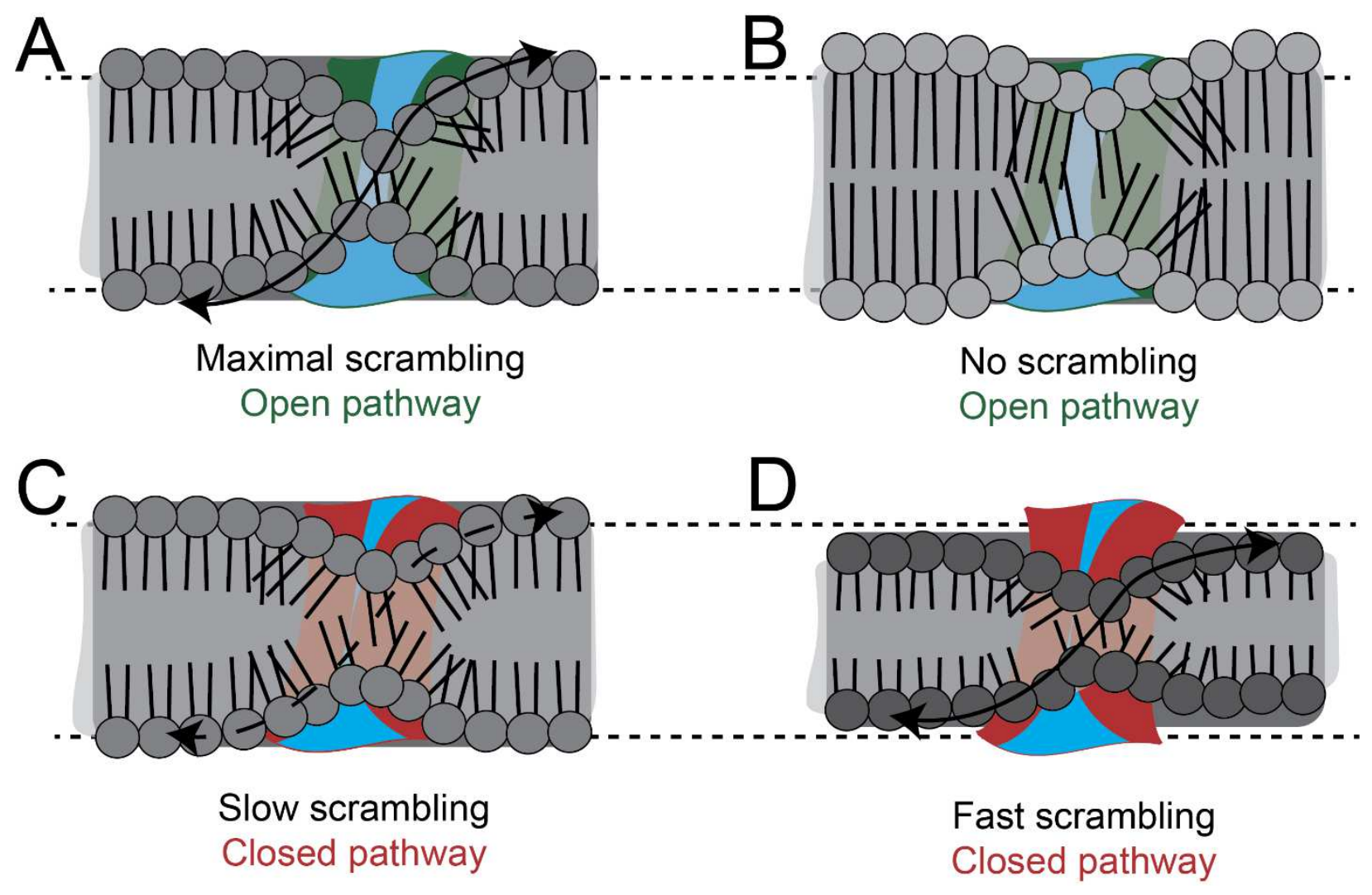

Figure 7. TMEM16 scramblases enable scrambling by thinning the membrane. A-D: Schematic representation of the open (A-B, colored in green) and closed pathways (C-D, colored in red) in membranes of different thickness. Cyan denotes regions accessible to water. Arrows denote high (solid line), low (dashed line) or no (no line) scrambling.

In sum, our results support a mechanism where during scrambling, lipids interact with the surface of the groove without having to penetrate and interact with its narrow and hydrophilic interior. Scrambling by the TMEM16's is modulated by two signals, binding of $\mathrm{Ca}^{2+}$ facilitates opening of the groove while the properties of the membrane determine whether the scramblase can thin the membrane enough to enable lipid flipping. This dual control of scrambling activity, by ligand binding and membrane properties, could constitute a secondary layer of regulation that 
prevents undesired lipid flipping by the TMEM16's during fluctuations in cellular cytosolic $\mathrm{Ca}^{2+}$ levels or when family members that reside in intracellular membranes are transiently localized to the plasma membrane. Similarly, rigidifying or thickening bilayer constituents, such as cholesterol, could silence the scrambling activity of other scramblases such as GPCR's in cellular membranes. 


\section{Data Availability}

All constructs are available on request. All models and associated cryoEM maps have been deposited into the Electron Microscopy Data Bank (EMDB) and the Protein Data Bank (PDB). The depositions include final maps, unsharpened maps, and associated FSC curves.

\begin{tabular}{|l|l|l|}
\hline Structure & PDB & EMDB \\
\hline $\mathrm{C} 18 / \mathrm{Ca}^{2+}$ dimer & $7 R X H$ & EMD-24730 \\
\hline $\mathrm{C} 18 / \mathrm{Ca}^{2+}$ monomer & $7 \mathrm{RXG}$ & EMD-24731 \\
\hline $\mathrm{C} 22 / \mathrm{Ca}^{2+}$ MSP1E3 & 7RX2 & EMD-24722 \\
\hline $\mathrm{C} 22 / \mathrm{Ca}^{2+}$ MSP2N2 & $7 \mathrm{RWJ}$ & EMD-24717 \\
\hline $\mathrm{C} 18 / 0 \mathrm{Ca}^{2+}$ & $7 \mathrm{RXB}$ & EMD-24727 \\
\hline $\mathrm{C} 14 / 0 \mathrm{Ca}^{2+}$ & $7 \mathrm{RX} 3$ & EMD-24723 \\
\hline $\mathrm{D} 511 \mathrm{~A} / \mathrm{E} 514 \mathrm{~A} \mathrm{C14/Ca^{2+ }}$ & 7RXA & EMD-24726 \\
\hline
\end{tabular}

Table 5: Data Availability

\section{Acknowledgements}

The authors thank members of the Accardi lab and Richard Hite for helpful discussions. The work was supported by National Institutes of Health (NIH) Grant R01GM106717 (to A.A.), by a Margaret and Herman Sokol Fellowship from Weill Cornell Medicine (M.E.F.), by the KBRI Basic Research Program through Korea Brain Research Institute funded by Ministry of Science and ICT (21-BR-01-08 to B.-C. L.) and National Research Foundation of Korea (NRF) grant funded by the Korea government (MSIT) (2019R1C1C1002699 to B.-C. L.). Some of this work was performed at the Simons Electron Microscopy Center and National Resource for Automated Molecular Microscopy located at the New York Structural Biology Center, supported by grants from the Simons Foundation (SF349247), NYSTAR, and the NIH National Institute of General Medical Sciences (GM103310). Part of the work was performed at the Cryo-EM Core Facility at University of Massachusetts Chan Medical School with the help of Dr. Kangkang Song and Dr. Chen Xu. Initial screening was performed at NYU Langone Health's Cryo-Electron Microscopy Laboratory (RRID: SCR_019202). 


\section{Author contributions}

M.F., Z.F. and A.A. designed the experiments; M.F., Z.F., O.E.A., Y.P. and B.-C.L. performed experiments; M.F., Z.F., O.E.A., Y.P., B.-C.L., X.C., E.F., S.S. and A.A. analyzed the data; M.F., S.S. and A.A. wrote the paper. All authors edited the manuscript.

\section{Competing Interests statement}

The authors declare no competing interests. 


\section{References}

1 Nagata, S., Sakuragi, T. \& Segawa, K. Flippase and scramblase for phosphatidylserine exposure. Current Opinion in Immunology 62, 31-38, doi:https://doi.org/10.1016/j.coi.2019.11.009 (2020).

2 Whitlock, J. M. \& Hartzell, H. C. Anoctamins/TMEM16 Proteins: Chloride Channels Flirting with Lipids and Extracellular Vesicles. Annu Rev Physiol 10, 119-143 (2017).

3 Suzuki, J., Umeda, M., Sims, P. J. \& Nagata, S. Calcium-dependent phospholipid scrambling by TMEM16F. Nature 468, 834-838 (2010).

4 Falzone, M., Malvezzi, M., Lee, B. C. \& Accardi, A. Known structures and unknown mechanisms of TMEM16 scramblases and channels. JGP 150, 933-947, doi:doi: 10.1085/jgp.201711957 (2018).

5 Kalienkova, V., Clerico Mosina, V. \& Paulino, C. The Groovy TMEM16 Family: Molecular Mechanisms of Lipid Scrambling and Ion Conduction. J Mol Biol, 166941, doi:10.1016/j.jmb.2021.166941 (2021).

6 Suzuki, J., Denning, D. P., Imanishi, E., Horvitz, H. R. \& Nagata, S. Xk-Related Protein 8 and CED-8 Promote Phosphatidylserine Exposure in Apoptotic Cells. Science 341, 403406, doi:10.1126/science.1236758 (2013).

7 Huang, F., Wong, X. \& Jan, L. Y. International Union of Basic and Clinical Pharmacology. LXXXV: calcium-activated chloride channels. Pharmacol Rev 64, 1-15 (2012).

8 Yang, H. et al. TMEM16F Forms a $\mathrm{Ca}(2+)$-Activated Cation Channel Required for Lipid Scrambling in Platelets during Blood Coagulation. Cell 151, 111-122 (2012).

9 Di Zanni, E., Gradogna, A., Scholz-Starke, J. \& Boccaccio, A. Gain of function of TMEM16E/ANO5 scrambling activity caused by a mutation associated with gnathodiaphyseal dysplasia. Cell Mol Life Sci 75, 1657-1670, doi:10.1007/s00018-0172704-9 (2018).

10 Gyobu, S. et al. A Role of TMEM16E Carrying a Scrambling Domain in Sperm Motility. Mol Cell Biol 36, 645-659, doi:10.1128/MCB.00919-15 (2015).

11 Vermeer, S. et al. Targeted next-generation sequencing of a $12.5 \mathrm{Mb}$ homozygous region reveals ANO10 mutations in patients with autosomal-recessive cerebellar ataxia. Am J Hum Genet 87, 813-819, doi:10.1016/j.ajhg.2010.10.015 (2010).

12 Paulino, C., Kalienkova, V., Lam, A. K. M., Neldner, Y. \& Dutzler, R. Activation mechanism of the calcium-activated chloride channel TMEM16A revealed by cryo-EM. Nature 552, 421-425, doi:10.1038/nature24652 (2017).

13 Brunner, J. D., Lim, N. K., Schenck, S., Duerst, A. \& Dutzler, R. X-ray structure of a calcium-activated TMEM16 lipid scramblase. Nature 516, 207-212 (2014).

14 Dang, S. et al. Cryo-EM structures of the TMEM16A calcium-activated chloride channel. Nature, doi:10.1038/nature25024 (2017).

15 Falzone, M. E. et al. Structural basis of $\mathrm{Ca} 2+$-dependent activation and lipid transport by a TMEM16 scramblase. eLife 8, e43229, doi:10.7554/eLife.43229 (2019).

16 Feng, S. et al. Cryo-EM Studies of TMEM16F Calcium-Activated Ion Channel Suggest Features Important for Lipid Scrambling. Cell Reports 28, 567-579.e564, doi:10.1016/j.celrep.2019.06.023 (2019).

17 Kalienkova, V. et al. Stepwise activation mechanism of the scramblase nhTMEM16 revealed by cryo-EM. eLife 8, e44364, doi:10.7554/eLife.44364 (2019).

18 Alvadia, C. et al. Cryo-EM structures and functional characterization of the murine lipid scramblase TMEM16F. eLife 8, e44365, doi:10.7554/eLife.44365 (2019). 
19 Khelashvili, G. et al. Dynamic modulation of the lipid translocation groove generates a conductive ion channel in Ca2+-bound nhTMEM16. Nature Communications 10, 4972, doi:10.1038/s41467-019-12865-4 (2019).

20 Bethel, N. P. \& Grabe, M. Atomistic insight into lipid translocation by a TMEM16 scramblase. Proc Natl Acad Sci USA 113, 14049-14054 (2016).

21 Suzuki, J. et al. Calcium-dependent phospholipid scramblase activity of TMEM16 protein family members. J Biol Chem 288, 13305-13316, doi:10.1074/jbc.M113.457937 (2013).

22 Segawa, K., Suzuki, J. \& Nagata, S. Constitutive exposure of phosphatidylserine on viable cells. PNAS 108, 19246-19251 (2011).

23 Bushell, S. R. et al. The structural basis of lipid scrambling and inactivation in the endoplasmic reticulum scramblase TMEM16K. Nature Communications 10, 3956, doi:10.1038/s41467-019-11753-1 (2019).

24 Malvezzi, M. et al. Out-of-the-groove transport of lipids by TMEM16 and GPCR scramblases. Proc Natl Acad Sci U S A 115, E7033-E7042, doi:10.1073/pnas.1806721115 (2018).

25 Lee, B.-C. et al. Gating mechanism of the extracellular entry to the lipid pathway in a TMEM16 scramblase. Nature Communications 9, 3251, doi:10.1038/s41467-018-057241 (2018).

26 Malvezzi, M. et al. Ca2+-dependent phospholipid scrambling by a reconstituted TMEM16 ion channel. Nature Communications 4, 2367, doi:10.1038/ncomms3367

https://www.nature.com/articles/ncomms3367\#supplementary-information (2013).

27 Brunner, J. D., Schenck, S. \& Dutzler, R. Structural basis for phospholipid scrambling in the TMEM16 family. Curr Opin Struct Biol 39, 61-70, doi:10.1016/j.sbi.2016.05.020 (2016).

28 Jiang, T., Yu, K., Hartzell, H. C. \& Tajkhorshid, E. Lipids and ions traverse the membrane by the same physical pathway in the nhTMEM16 scramblase. Elife 6, doi:10.7554/eLife.28671 (2017).

29 Griffin, D. A. et al. Defective membrane fusion and repair in Anoctamin5-deficient muscular dystrophy. Hum Mol Genet 25, 1900-1911, doi:10.1093/hmg/ddw063 (2016).

$30 \mathrm{Yu}, \mathrm{K}$. et al. Identification of a lipid scrambling domain in ANO6/TMEM16F. eLife 4, 123, doi:10.7554/eLife.06901 (2015).

31 Peters, C. J. et al. The Sixth Transmembrane Segment Is a Major Gating Component of the TMEM16A Calcium-Activated Chloride Channel. Neuron 97, 1063-1077.e1064, doi:https://doi.org/10.1016/j.neuron.2018.01.048 (2018).

32 Le, T. et al. An inner activation gate controls TMEM16F phospholipid scrambling. Nature Communications 10, 1846, doi:10.1038/s41467-019-09778-7 (2019).

33 Pomorski, T. \& Menon, A. K. Lipid flippases and their biological functions. Cell Mol Life Sci 63, 2908-2921 (2006).

34 Menon, I. et al. Opsin is a phospholipid flippase. Current Biology 21, 149-153, doi:10.1016/j.cub.2010.12.031 (2011).

35 Straub, M. S., Alvadia, C., Sawicka, M. \& Dutzler, R. Cryo-EM structures of the caspaseactivated protein XKR9 involved in apoptotic lipid scrambling. eLife 10, e69800, doi:10.7554/eLife.69800 (2021).

36 Sakuragi, T. et al. An intramolecular scrambling path controlled by a gatekeeper in $\mathrm{Xkr8}$ phospholipid scramblase. bioRxiv, 2021.2005.2006.442885, doi:10.1101/2021.05.06.442885 (2021). 
37 Khelashvili, G. et al. Membrane lipids are both the substrates and a mechanistically responsive environment of TMEM16 scramblase proteins. Journal of computational chemistry 41, 538-551, doi:10.1002/jcc.26105 (2020).

38 Goren, M. A. et al. Constitutive phospholipid scramblase activity of a $\mathrm{G}$ protein-coupled receptor. Nat Commun 5, 5115, doi:10.1038/ncomms6115 (2014).

39 Yu, K., Duran, C., Qu, Z., Cui, Y.-Y. \& Hartzell, H. C. Explaining Calcium-Dependent Gating of Anoctamin-1 Chloride Channels Requires a Revised Topology. Circulation Research 110, 990-999, doi:10.1161/CIRCRESAHA.112.264440 (2012).

40 Tien, J. et al. A comprehensive search for calcium binding sites critical for TMEM16A calcium-activated chloride channel activity. Elife, e02772, doi:10.7554/eLife.02772 (2014).

41 Suzuki, J., Imanishi, E. \& Nagata, S. Xkr8 phospholipid scrambling complex in apoptotic phosphatidylserine exposure. Proc Natl Acad Sci USA 113, 9509-9514 (2016).

42 Watanabe, R., Sakuragi, T., Noji, H. \& Nagata, S. Single-molecule analysis of phospholipid scrambling by TMEM16F. Proceedings of the National Academy of Sciences 115, 3066-3071, doi:10.1073/pnas.1717956115 (2018).

43 Stansfeld, P. J. et al. MemProtMD: automated insertion of membrane protein structures into explicit lipid membranes. Structure 23, 1350-1361, doi:10.1016/j.str.2015.05.006 (2015).

44 Kostritskii, A. Y. \& Machtens, J.-P. Molecular mechanisms of ion conduction and ion selectivity in TMEM16 lipid scramblases. Nature Communications 12, 2826, doi:10.1038/s41467-021-22724-w (2021).

45 Stix, R., Song, J., Banerjee, A. \& Faraldo-Gómez, J. D. DHHC20 Palmitoyl-Transferase Reshapes the Membrane to Foster Catalysis. Biophys $J$ 118, 980-988, doi:10.1016/j.bpj.2019.11.003 (2020).

46 Parsegian, A. Energy of an ion crossing a low dielectric membrane: solutions to four relevant electrostatic problems. Nature 221, 844-846, doi:10.1038/221844a0 (1969).

47 Bennett, W. F. D. \& Tieleman, D. P. The Importance of Membrane Defects-Lessons from Simulations. Accounts of Chemical Research 47, 2244-2251, doi:10.1021/ar4002729 (2014).

48 Wang, L. et al. Scrambling of natural and fluorescently tagged phosphatidylinositol by reconstituted G protein-coupled receptor and TMEM16 scramblases. Journal of Biological Chemistry 293, 18318-18327, doi:10.1074/jbc.RA118.004213 (2018). 


\begin{tabular}{|c|c|c|c|}
\hline Name & $\begin{array}{c}\text { Lipid Component } \\
\text { Chain Length } \\
\text { (70\% PC:30\%PG) }\end{array}$ & $\begin{array}{c}\text { Height from } \\
\text { AFM (nm) }\end{array}$ & $\begin{array}{c}\text { Height estimated } \\
\text { from EM maps } \\
(\mathrm{nm})\end{array}$ \\
\hline C14 & $\begin{array}{c}50 \% 14: 0,50 \% \\
16: 0-18: 1\end{array}$ & $3.2 \pm 0.29$ & $3.0 \pm 1.5$ \\
\hline C16 & $100 \% 16: 1$ & $3.2 \pm 0.22$ & N.d. \\
\hline \multirow[t]{2}{*}{ C18 } & \multirow[t]{2}{*}{$100 \% 18: 1$} & \multirow[t]{2}{*}{$3.4 \pm 0.19$} & $\begin{array}{l}\left(0 \mathrm{Ca}^{2+}\right) \\
3.1 \pm 3.5\end{array}$ \\
\hline & & & $\begin{array}{c}\left(0.5 \mathrm{mM} \mathrm{Ca}^{2+}\right) \\
3.3 \pm 0.95\end{array}$ \\
\hline $\mathrm{C} 20$ & $100 \% 20: 1$ & $3.7 \pm 0.22$ & N.d. \\
\hline $70 \% \mathrm{C} 22$ & $\begin{array}{l}70 \% 22: 1, \\
30 \% 18: 1\end{array}$ & $4.0 \pm 0.26$ & N.d. \\
\hline $70 \%$ C22 & $\begin{array}{l}70 \% 22: 1 \text { PC, } \\
30 \% 18: 1 \text { PG }\end{array}$ & $4.0 \pm 0.19$ & N.d. \\
\hline $\mathrm{C} 22$ & $100 \% 22: 1$ & $4.1 \pm 0.19$ & $3.9 \pm 0.85$ \\
\hline
\end{tabular}

Table 1: AFM and cryoEM determination of membrane thickness for considered lipid compositions. Heights were estimated using AFM tomography, reported values indicate the peak $\mathrm{FWHH} \pm$ of the value distribution (see Supp. Fig. 4A-B and Methods). For cryoEM membrane height was determined from $\mathrm{C} 1$ unsharpened EM maps using the difference in $\mathrm{z}$ coordinate for the inner and outer leaflet at $(\mathrm{x}, \mathrm{y})$ far from the protein. Reported values are the average $\pm \mathrm{S}$.Dev of 3 different points. N.d. indicates compositions for which no cryoEM data was determined. 


\section{Supplementary Files}

This is a list of supplementary files associated with this preprint. Click to download.

- FalzoneetalSI.pdf

- FalzoneetalMethods.pdf

- Falzonenrreportingsummary.pdf

- nreditorialpolicychecklist.pdf

- FalzoneetalValidationReports.pdf 